\title{
Wheeze monitoring in children for assessment of nocturnal asthma and response to therapy
}

\author{
L. Bentur*,\#, R. Beck*, M. Shinawi*, T. Naveh*, N. Gavriely
}

Wheeze monitoring in children for assessment of nocturnal asthma and response to therapy. L. Bentur, R. Beck, M. Shinawi, T. Naveh, N. Gavriely. (C) ERS Journals Ltd 2003.

ABSTRACT: The utilisation of nocturnal wheeze monitoring and quantification for assessment of asthma activity was studied in symptomatic school-aged children before and during treatment.

Twelve children 6-14 yrs of age with mild or moderate untreated asthma were studied at home three times: before, $48 \mathrm{~h}$ and 6 weeks into treatment with $5 \mathrm{mg}$ montelukast daily. Lung sounds were recorded overnight by an automatic wheeze detection device (PulmoTrack $(B)$ ). Per cent wheezing within each respiratory cycle was calculated every $30 \mathrm{~s}$ throughout the night and a Nocturnal Wheeze Index (NWI) was calculated for the total night. The results were compared with spirometric indices (forced expiratory volume in one second (FEV1), forced vital capacity), bronchial reactivity (provocative concentration causing a $20 \%$ fall in FEV1 by adenosine $5^{\prime}$-monophosphate (PC20)) and daily symptom scores, performed in parallel at each stage of the study.

The pretreatment NWI was $814 \pm 898$ (mean \pm SD), which declined to $318 \pm 1992$ days after onset, and to $137 \pm 101$ after 6 weeks of treatment. The NWI in seven healthy children was $47 \pm 43$. The FEV1, PC20 and symptom scores improved in parallel.

Wheeze monitoring provides quantitative and noninvasive information about the extent of nocturnal wheezing in children, correlates well with conventional indices of asthma activity and can assist in assessing efficacy of treatment.

Eur Respir J 2003; 21: 621-626.
*Division of Paediatric Pulmonology, Rambam Medical Centre and ${ }^{\#}$ Pulmonary Physiology Section, The Bruce Rappaport Faculty of Medicine at Technion - Israel Institute of Technology, Haifa, Israel.

Correspondence: N. Gavriely, Pulmonary Physiology Section, The Bruce Rappaport Faculty of Medicine, Technion - Israel Institute of Technology, P.O. Box 9697, Haifa 31096, Israel. Fax: 97248533185

E-mail: gavriely@tx.technion.ac.il

Keywords: Asthma monitoring, automatic wheeze detection, breath sounds, leukotriene receptor antagonist, nocturnal asthma, pulmonary acoustics

Received: May 12002

Accepted after revision: November 112002

This study was funded in part by grants from Merck, Sharp \& Dohme (MSD), Israel and by Karmel Medical Acoustic Technologies Ltd, Yokneam Illit, Israel.
Worsening of asthma symptoms at night is a common, troublesome, yet poorly recognised component of the disease. Nocturnal asthma (NA) symptoms were reported in $47-75 \%$ of asthma patients in a number of large surveys from different countries $[1,2]$. Patients with NA often do not wake up until their airways are severely obstructed. The need to recognise and treat NA is also based on the observation that improvement in sleep quality and daytime psychological function, including resolution of learning and behavioural difficulties, occurs when patients with NA (adults and children) are treated successfully $[3,4]$.

Objective measurements of airflow obstruction during sleep are difficult to obtain. A diurnal variation of $>15 \%$ in forced expiratory volume in one second (FEV1) or peak expiratory flow (the "morning dip") and symptom diaries have been used as a quantitative and objective, yet indirect, method to detect the presence and severity of NA. The FEV1 of NA patients was $31 \%$ lower early in the morning than in the previous afternoon [5], but no detectable changes in the respiratory rate or expiratory duration were found during sleep. Direct measurement of airway resistance during sleep requires substantial patient instrumentation and cannot be used in routine clinical practice. When studies of NA patients did include these measurements, a pattern of substantially increased airflow obstruction during sleep was found [6]. The need for a simple, objective and quantitative method to assess NA severity is illustrated by the study of FALCONER et al. [7], who found poor agreement between subjective estimation and objective measurements of cough, a common symptom of NA.

Wheezing is the acoustic manifestation of airways obstruction
[8] and can be recorded and monitored noninvasively with minimal patient cooperation during sleep or wakefulness. An automatic wheeze detection device was used by LENCLUD et al. [9] to compare tracheal wheezes with measurements of airway resistance. A $79 \%$ positive predictive power and $83 \%$ negative predictive power were reported with this system. In another study, KIYOKAWA et al. [10] recorded tracheal sounds in asthmatic patients during sleep, before and after a change in treatment. Observers performed auditory review and manually recorded the presence of wheezes. Although tedious, this method provided objective information on the response to therapy.

The authors hypothesised that quantification of wheezes can provide accurate information on NA activity and would correlate with other measures of asthma severity. The use of a

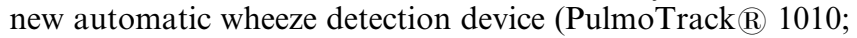
Karmel Medical Acoustic Technologies Ltd, Yokneam Illit, Israel) for all-night monitoring of asthmatic children and healthy controls is described.

\section{Methods}

\section{Study design}

This was a prospective pilot study with each subject serving as their own control. Rambam Medical Centre's Institutional Review Board approved this study. All procedures were explained to the children and the parents signed a detailed informed consent form. 


\section{Patients}

Untreated asthmatic children referred to a paediatric pulmonary clinic were recruited. Inclusion criteria were as follows: 1) age 6-14 yrs; 2) diagnosis of asthma by Global Initiative for Asthma (GINA) criteria [11]; 3) ability to perform spirometry consistently; 4) FEV1 $>60 \%$ predicted at initial assessment; and 5) asthma severity "mild persistent" or "moderate persistent" by GINA classification [11]. Exclusion criteria were as follows: 1) other chronic conditions; 2) emergency room visit in the previous 3 months; 3 ) respiratory infection in the previous month; and 4) use of anti-inflammatory medication in the previous 3 months, or any asthma medication except short-acting bronchodilators in the 2 weeks prior to enrolment. The healthy subjects were children who were never diagnosed with any respiratory disorder, had normal spirometry and a normal physical exam.

\section{Study procedure}

Untreated children with symptomatic asthma were enrolled and treated with montelukast to control their symptoms. Nocturnal wheezing, conventional asthma indices and symptom scores were measured during therapy. At enrolment, inclusion/exclusion criteria were verified, baseline spirometry was performed, and a 2-week run-in period was started, during which the subjects used short-acting inhaled bronchodilators only and completed a daily symptom diary. At the end of the 2-week run-in period, overnight acoustic respiratory monitoring (ARM) of wheezing activity was performed for wheeze quantification. Spirometry and an adenosine 5'-monophosphate (AMP) bronchial provocation test (BPT) were also performed, prior to which the children were examined by stethoscope and a 10-min ARM performed to detect presence of wheezes. Five milligrams daily montelukast (Singulair $^{\mathrm{TM}}$, Merck Sharp \& Dome Ltd, Israel), an oral leukotriene receptor antagonist, was then started at bedtime. ARM, spirometry and AMP BPT, as described above, were repeated 2 days later to assess the early effects of montelukast (onset), and after 6 weeks to assess the delayed effects (during).

\section{Description of acoustic respiratory monitoring procedure}

Recording and analysis of respiratory sounds were conducted according to standardised methods as described previously $[12,13]$. Respiratory acoustic signals were recorded from five phonopneumography piezoelectric contact sensors (PPG Sensors; Karmel Medical Acoustic Technologies Ltd) applied over the trachea, right and left axillae and both posterior bases of the lungs. The sensors were coin-shaped piezoelectric elements with linear $\pm 3 \mathrm{~dB}$ frequency response of $75-2,000 \mathrm{~Hz}$, a resonance at $2.7 \mathrm{kHz}$, a useable range that extended beyond $4 \mathrm{kHz}$ and a built-in passive ambient noise rejection capability. The sensors were attached to the chest with adhesive foam pads that further reduced ambient noise interference and eliminated contact noise. All sensors were

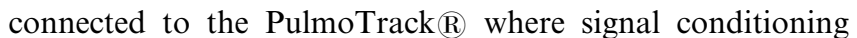
(amplification $\times 3,000$, band pass filtration $80-4,000 \mathrm{~Hz}$ at $24 \mathrm{~dB} /$ oct) was performed prior to analogue-to-digital conversion $\left(11,025\right.$ samples $\cdot \mathrm{s}^{-1} \cdot$ channel $\left.^{-1}\right)$. Two other signals were tracked; ambient noise, with an air-coupled microphone placed near the patient, and chest impedance for measurement of breathing activity (respiratory rate, phase and amplitude).

Prior to sleep, the sensors were placed by a technician who monitored the procedure for the entire night and noted any unusual events. The sensors and wires posed no restriction on the children, who moved freely during sleep.
Wheezing was continuously measured at the five sensor positions simultaneously using all data received from the sensors and was quantified in real time as per cent of wheezing time to breathing time, wheeze rate $\left(\mathrm{Wz}^{\%}\right)$ [14]. Wheeze detection was performed by a fast Fourier transform-based algorithm that was previously verified and found to have sensitivity of $91 \%$ and specificity of $89 \%$ when compared with consensus assessment by a panel of experts [15]. Speech, crying and other vocal cord sounds are identified by the system and discarded [15]. In addition, auditory audit of the data was also performed to verify the detection accuracy. The detected wheezes are shown continuously as a Wheezogram $\mathbb{R}$, (Karmel Medical Acoustic Technologies Ltd) a plot of wheeze frequency versus time (fig. 1), along with the chest impedance signal. In addition, an Acoustic Vector ${ }^{\mathrm{TM}}$ (Karmel Medical Acoustic Technologies Ltd) continuously shows the timing of wheezes

a)

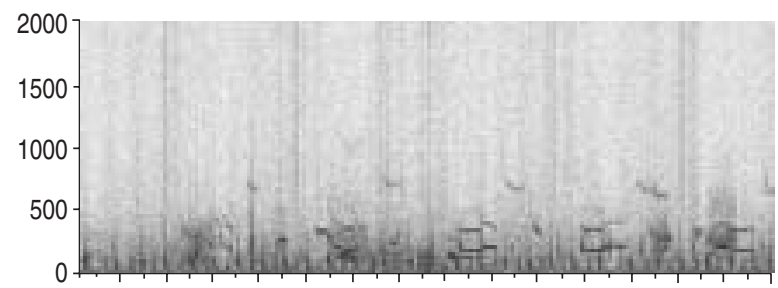

b)
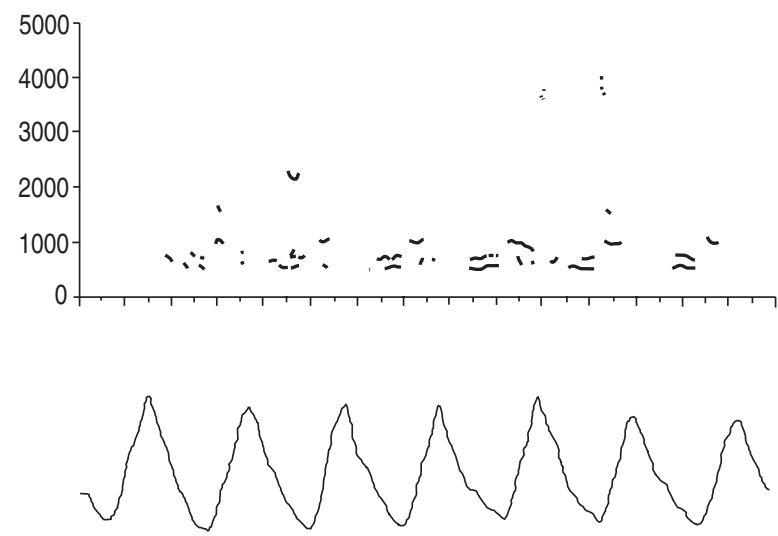

d)

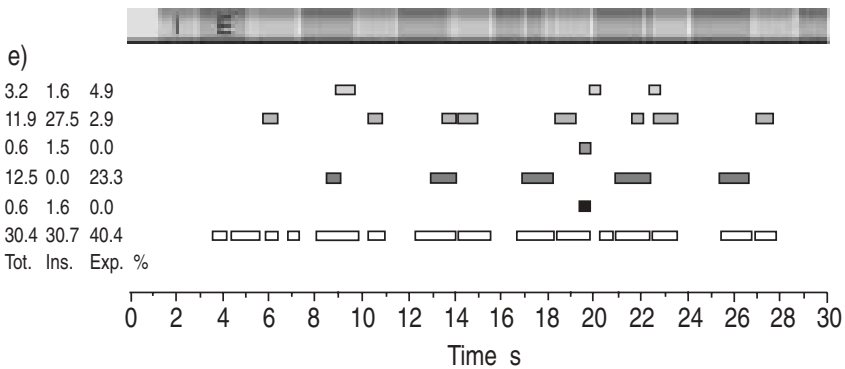

Fig. 1.-Example of a 30-s data segment from patient Respiratory Monitoring Study number 3. a) Sonogram plot of frequency of wheezes in $\mathrm{Hz}$ versus time with amplitude in grey scale shown for the left axilla channel. b) Wheezogram $\mathbb{R}$ plot showing the wheezes detected by the automatic wheeze detection algorithm in all five channels, which are displayed individually as indicated on the left of e). c) Breathing activity by chest impedance. Respiratory rate: 14.1 breaths per min; inspiratory duration: $1.5 \mathrm{~s}$; expiratory duration: $2.7 \mathrm{~s}$; inspiration:expiration: 0.6. d) Detection of inspiratory $(\mathrm{I} ; \mathrm{D})$, and expiratory (E; $\square)$ phases. e) Acoustic vector plot, indicating the timing of wheeze activity in the different channels (first row: tracheal whistles; second row: tracheal wheezes; third row: right base; fourth row: left base; fifth row: right axilla; sixth row: left axilla). Ambient channel showed no evidence of wheezing (not shown). The total (Tot.), inspiratory (Ins.) and expiratory (Exp.) wheeze rates \% are shown. 

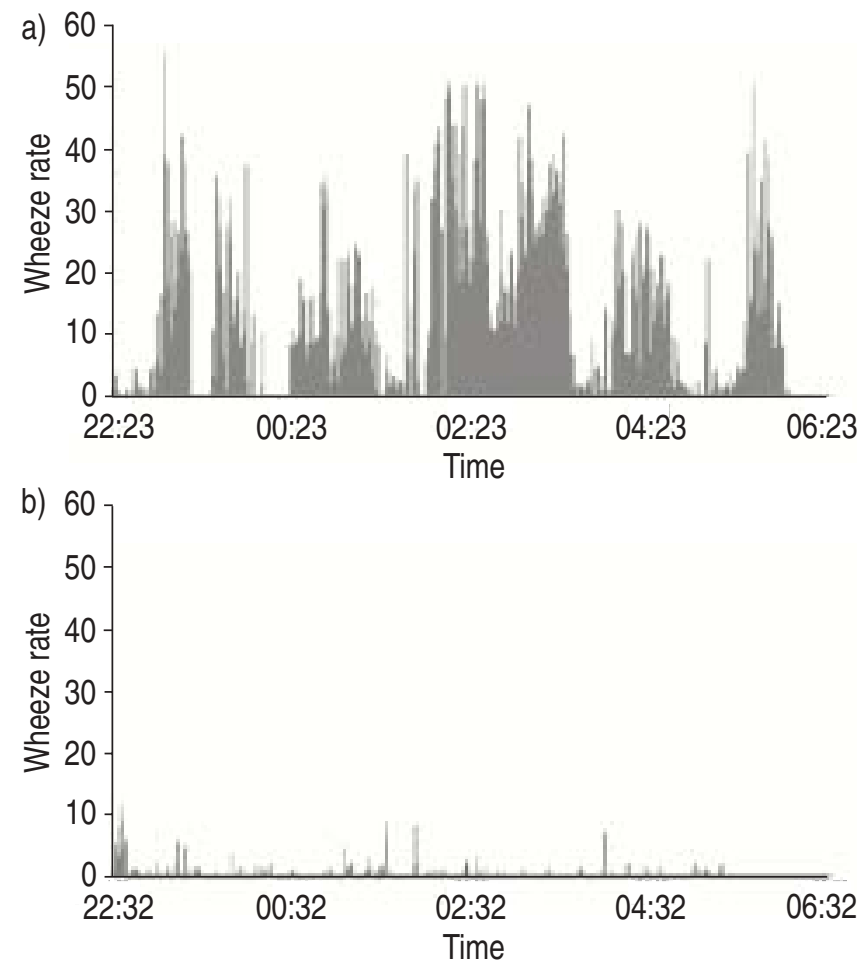

Fig. 2.-Wheeze activity during a full night's sleep in a 9-yr-old asthmatic patient (Respiratory Monitoring Study number 2) a) before treatment and b) 2 days after starting treatment. Each narrow bar represents the combined per cent time wheezing (wheeze rate) from all channels in a 30-s sound segment. Note periods of significant wheezing throughout the night in a) and their marked diminution in b). The patient did not wake up during both nights.

detected at the different sensor sites. The automatic wheeze detection algorithm identifies continuous adventitious breath sounds at $80-4,800 \mathrm{~Hz}$ in the tracheal channel and $80-2,400 \mathrm{~Hz}$ in the chest wall channels. These frequency ranges include low-frequency wheezes and rhonchi as well as high-pitch tracheal "whistles" [16].

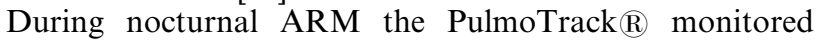
wheezing activity throughout the night $(\sim 8 \mathrm{~h})$ and logged the timing and frequency of all detected wheezes, as well as information on ambient noise and breathing activity. In addition, the system automatically recorded 30-s segments of raw data every $10 \mathrm{~min}$, for quality assurance and possible further future analyses.

\section{Data reduction and statistical methods}

At the conclusion of the nocturnal recording, the wheeze rate $\left(\mathrm{Wz}^{\circ} \%\right)$ was displayed for each channel in successive 30 -s intervals. The full night's data were plotted as a trend report as shown in figure 2. These data can be plotted for any one of the five sensors or combination of sensors and by respiratory phase. The trend report provides a temporal distribution of wheezing activity during the night. The system also provides a quantitative numeric output in the form of a distribution function (histogram) of the $\mathrm{Wz} \%$ as the rate of occurrence (in $\%$ of the total night test) of wheeze activity in each of the following $\mathrm{Wz} \%$ ranges: $0.0-0.75,0.76-1.50,1.51-3.00,3.01-6.00$, $6.01-12.00,12.01-24.00,24.01-48.00$ and $48.01-100.0$ (fig. 3). Integrating the total wheeze activity, analogous to calculation of the area under the curve, reduced the data further. This was done by multiplying the "rate of occurrence" for each $\mathrm{Wz}^{\%} \%$ range by the mean of that range and summing the total for all
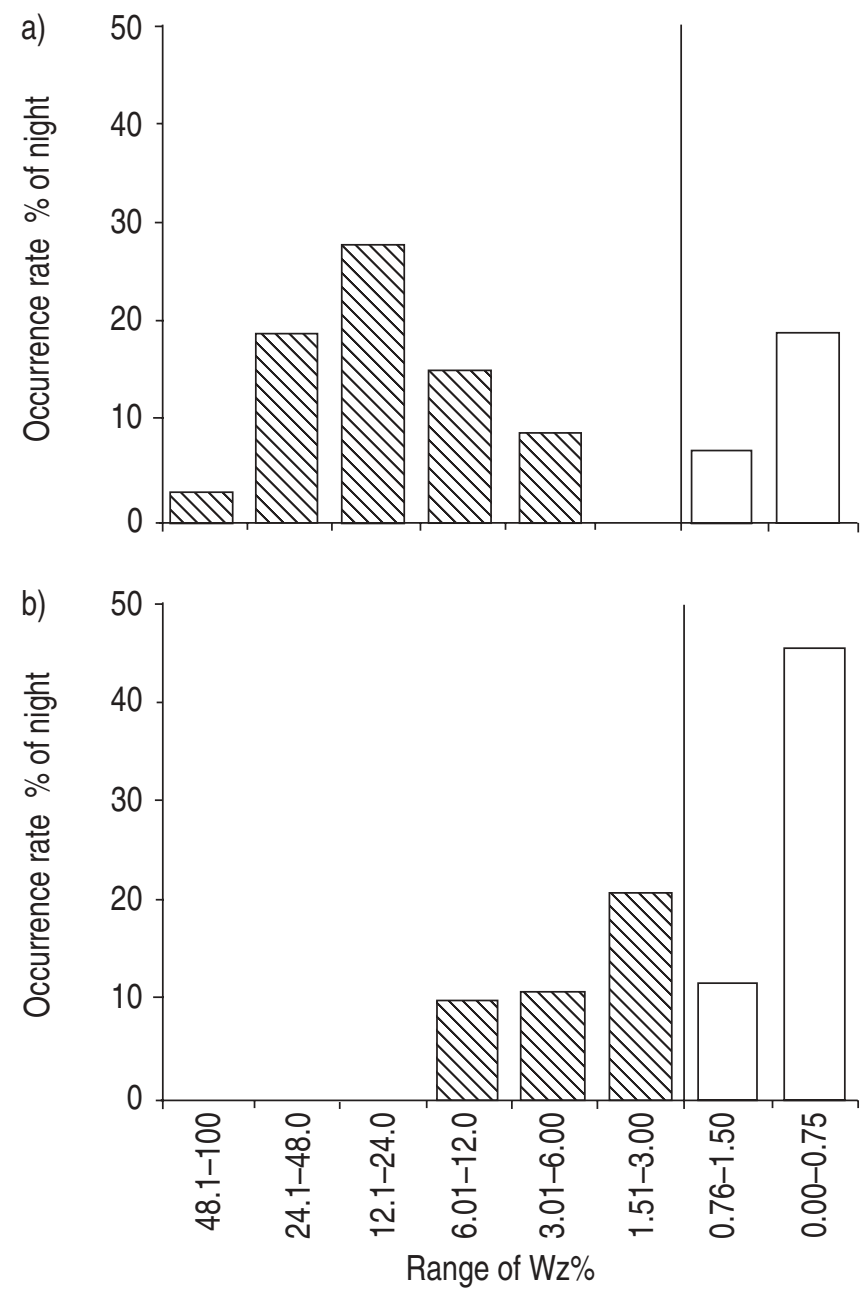

Fig. 3.-Examples of wheeze activity histograms a) before and b) during treatment with montelukast. Occurrence rate is the per cent of the total number of 30 -s segments with wheeze rate $\left(\mathrm{Wz}^{\circ} \%\right)$ that fall within the predetermined ranges shown on the ordinate. $\mathrm{Wz} \%$ values $<1.5 \%(\square)$ were considered within normal range, $\mathrm{Wz} \%>1.5 \%$ (\$) above normal range. Total extent of wheezing a) 229, 678, 505, 140,42 , and b) $88,52,48$ from left to right, is calculated from the bar size and the middle value of the $\mathrm{Wz} \%$ range.

the ranges. This calculated the nocturnal wheeze index (NWI). Accordingly, the maximal possible NWI score is 7,400 .

\section{Establishing normal range}

This study conducted ARM in healthy children that had never been diagnosed with any respiratory disorders and had normal physical examination and normal spirometry. The monitoring procedure was identical to that of the study group and was done on two consecutive nights to establish intrasubject variability.

\section{Adenosine bronchial provocation}

BPT was performed by inhalation of AMP solutions in doubling doses [17]. Fresh solutions of AMP (SIGMA, St. Louis, MO, USA) were prepared, starting at $0.39 \mathrm{mg} \cdot \mathrm{mL}^{-1}$ to a maximum of $400 \mathrm{mg} \cdot \mathrm{mL}^{-1}$. Nebulised AMP solution was inhaled for $2 \mathrm{~min}$, followed by spirometry (Microloop II portable spirometer; Micromedical Ltd, Rochester, Kent, England). 
Table 1. - Patient baseline data and changes in forced expiratory volume in one second (FEV 1$)$, forced vital capacity (FVC) and provocation dose causing a $20 \%$ fall in $\mathrm{FEV}_{1}$ (PC20; expressed as the adenosine $5^{\prime}$-monophosphate (AMP) doubling dose number at which FEV 1 dropped by $20 \%$ ) during the various study phases. Wilcoxon tests were used to compare the study phases

\begin{tabular}{|c|c|c|c|c|c|c|c|c|c|c|c|c|c|}
\hline \multirow[t]{2}{*}{ Patient } & \multirow{2}{*}{$\begin{array}{l}\text { Test } \\
\text { age }\end{array}$} & \multicolumn{3}{|c|}{ FEV1 \% pred } & \multicolumn{3}{|c|}{ FVC $\%$ pred } & \multicolumn{3}{|c|}{ PC20 AMP doubling dose } & \multicolumn{3}{|c|}{ Combined symptom score } \\
\hline & & Pre- & Onset & During & Pre- & Onset & During & Pre- & Onset & During & Pre- & Onset & During \\
\hline 1 & 9 & 58 & 61 & 74 & 73 & 74 & 85 & 5 & 4 & 8 & 23 & 14 & 7 \\
\hline 2 & 6 & 82 & 94 & 91 & 95 & 90 & 102 & 3 & 6 & 7 & 44 & 18 & 8 \\
\hline 3 & 10 & 70 & 73 & 78 & 82 & 78 & 81 & 2 & 5 & 6 & 30 & 9 & 10 \\
\hline 4 & 10 & 79 & 82 & 85 & 89 & 90 & 92 & 10 & 10 & 12 & 11 & 3 & 0 \\
\hline 5 & 8.5 & 90 & 88 & 100 & 95 & 90 & 100 & 8 & 12 & 12 & 46 & 12 & 7 \\
\hline 6 & 6 & 89 & 89 & 94 & 83 & 86 & 87 & 7 & 6 & 6 & 67 & 29 & 11 \\
\hline 7 & 9 & 62 & 62 & 68 & 70 & 66 & 75 & 2 & 4 & 5 & 36 & 11 & 10 \\
\hline 8 & 14 & 82 & 85 & 87 & 88 & 92 & 88 & 5 & 8 & 5 & 49 & 20 & 17 \\
\hline 9 & 9 & 73 & 75 & 77 & 82 & 80 & 80 & 3 & 7 & 7 & & & \\
\hline 10 & 13 & 69 & 72 & 85 & 74 & 69 & 82 & 6 & 5 & 6 & 23 & 6 & 0 \\
\hline 11 & 6 & 70 & 90 & 97 & 60 & 76 & 87 & 4 & 4 & 5 & 18 & 1 & 2 \\
\hline 12 & 14 & 91 & 85 & 90 & 99 & 95 & 98 & 6 & 6 & 9 & 25 & 15 & 3 \\
\hline Mean & 6.5 & 76.3 & 79.7 & 85.5 & 82.5 & 82.2 & 88.0 & 5.1 & 6.4 & 7.5 & 33.8 & 12.5 & 6.8 \\
\hline $\mathrm{SD}$ & 2.9 & 11.0 & 10.9 & 9.8 & 11.6 & 9.6 & 8.5 & 2.5 & 2.5 & 2.5 & 16.4 & 8.0 & 5.2 \\
\hline \multirow[t]{2}{*}{$\mathrm{p}$-value } & & & $\mathrm{NS}^{\#}$ & $0.005^{\circ}$ & & $\mathrm{NS}^{\#}$ & $0.004^{\top}$ & & $0.043^{\#}$ & NS & & $0.001^{\#}$ & $0.01^{\bullet}$ \\
\hline & & & & $0.003^{\#}$ & & & $0.03^{\#}$ & & & $0.0001^{\#}$ & & & $0.001^{\#}$ \\
\hline
\end{tabular}

Statistical analysis comparing the study phases (Wilcoxon) is shown. \% pred: \% predicted; NS: nonsignificant. ${ }^{\#}$ : compared with the prestudy phase; ${ }^{\uparrow}$ : compared with the onset study phase.

The best of three efforts was recorded. The provocation dose was doubled at 5-min intervals until the FEV1 dropped $20 \%$ below baseline level (PC20), or a concentration of $400 \mathrm{mg} \cdot \mathrm{mL}^{-1}$ was reached, producing a negative result.

\section{Symptom scores}

The subjects completed a daily asthma symptom diary that included day cough, night cough, exercise tolerance and the use of bronchodilators. Each category was graded as none (0), mild (1) or significant (2). Combined scores of the four categories for 2-week periods (maximal possible score 112), corresponding to the "pre", "onset" and "during" stages, were tallied and compared.

\section{Statistical analysis}

Nonparametric Wilcoxon rank-sum test was used to analyse the changes of the various parameters in the different stages of the study. A p-value $<0.05$ was considered significant.

\section{Results}

Sixteen asthmatic children were enrolled into the study and 12 (six males and six females) completed it. Their ages ranged from 6-14 yrs (mean 9.5). Four patients dropped out, two due to intercurrent illness (viral meningitis, pneumonia) and two for personal reasons. Seven healthy children had nocturnal ARM performed, five of them on two consecutive nights. The results of the changes in the various parameters during the three study phases are presented in table 1 and in figure 4.

\section{Acoustic respiratory monitoring data}

All ARM sessions were completed without incident, the children slept normally and were not bothered by the attached sensors. A Wheezogram $(\mathrm{R}$ from one of the patients Respiratory Monitioring Study (RMS) number 3) is shown in figure 1. All children where wheeze-free when examined at
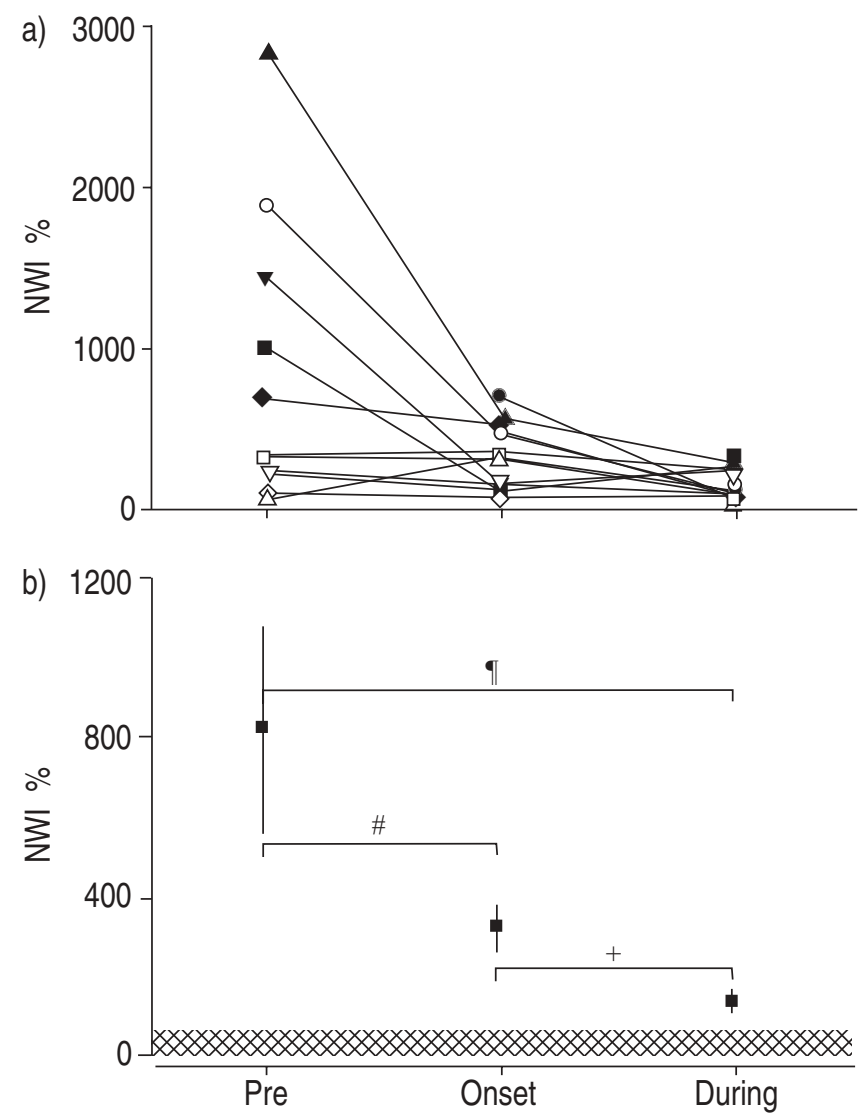

Fig. 4. -a) Individual nocturnal wheeze index (NWI) values for all 12 study subjects at the three phases of treatment. b) Average \pm SEM values of NWI in the 12 study subjects and the corresponding values \pm SEM of the healthy group. E: normal range. ${ }^{\#}: \mathrm{p}=0.05$; ๆ: $\mathrm{p}=0.028 ;{ }^{+}: \mathrm{p}=0.026$. 
daytime prior to their spirometry and AMP BPT, both by stethoscope and a 10-min ARM.

\section{Nocturnal ARM data}

The $\mathrm{Wz} \%$ values from a full night of pretreatment monitoring of a 9-yr-old asthma patient (RMS number 2) are shown in figure 2a. Multiple substantial wheezing episodes lasting 30-60 min each that subsided and reappeared spontaneously without treatment and without waking up are evident. Wheezing activity diminished markedly after 2 days of treatment (onset), as shown in figure $2 \mathrm{~b}$. Examples of the wheeze activity distribution curve (histogram) with calculation of the NWI for patient RMS number 2 at the pre- and during stages are shown in figure 3 . The individual and mean \pm SD of the values of the NWI from the 12 patients who completed the protocol are shown in figure 4 . Note that in the six patients with mild persistent asthma and normal spirometry (numbers $2,4,5,6,8$, and 12 ; table 1 ), four had a high degree of nocturnal wheezing. In nine of the subjects $(75 \%)$ the NWI diminished substantially after 6 weeks of treatment. In the other three subjects $(25 \%)$, no changes in NWI were detected. The mean NWI fell six-fold between the pre- and the during stages $(\mathrm{p}=0.028)$. This fall consisted of 2.6-fold diminution after $48 \mathrm{~h}(\mathrm{p}=0.05)$ and additional 2.3-fold reduction after 6 weeks $(p=0.026)$. Auditory audit of the above data confirmed the accuracy of the automatic analysis performed by the

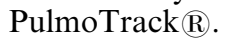

\section{Establishing normal range}

ARM was conducted in seven healthy children, five of whom had ARM on two consecutive nights to establish intrasubject variability. The healthy group's NWI was $47 \pm 43 \%$, with a range of 6-122. Intra-subject variability was small ( $\pm 10 \%$ maximum). The "normal" range is shown in figure $4 \mathrm{~b}$ as the shaded area representing the mean \pm SD of the healthy group.

Pre- to onset. Mean NWI fell from $814 \pm 898$ to $318 \pm 199$ $(\mathrm{p}=0.05)$. By doubling doses, there was a statistically significant improvement in PC20 by $1.3(\mathrm{p}=0.043)$, but no statistical change in FEV1 or FVC. In contrast, the symptom scores showed a marked and statistically significant improvement in all categories (table 1).

Pre- to during. There was further improvement from onset in all parameters after 6 weeks of treatment (table 1). Mean NWI fell further to $137 \pm 101$ ( $\mathrm{p}=0.028), \mathrm{PC} 20$ improved by one additional doubling dose to $7.5 \pm 2.5(\mathrm{p}=0.0001)$, FEV1 improved from $76.3 \pm 11.0$ to $85.5 \pm 9.8 \%$ pred $(\mathrm{p}=0.003)$ and $\mathrm{FVC}$ rose from $82.5 \pm 11.6$ to $88.0 \pm 8.5 \%$ pred ( $\mathrm{p}=0.03$ ). Symptom scores remained at the same low levels of onset, significantly better than prestudy (table 1).

\section{Discussion}

This study demonstrates that computerised nocturnal wheeze monitoring is a practical and noninvasive method for assessing asthma activity in children and that the results of nocturnal wheeze quantification correlate well with standard asthma indices such as spirometry results, PC20 and symptom scores.

Nocturnal wheezing as a manifestation of uncontrolled asthma is a well-known phenomenon. Previous studies have quantified nocturnal wheeze activity using automated or auscultatory methods to generate an objective estimate of the extent of wheezing $[9,10,14]$. An important finding of the present study was the large extent of nocturnal wheezing in the paediatric asthma patients. Based on their clinical symptoms and spirometric data, all patients who participated in this study were classified as having either mild persistent or moderate persistent asthma [11]. The fact that they were untreated may account for some increase in symptoms. However, most of the patients were unaware of nocturnal symptoms and they were categorised according to other symptoms and pulmonary functions. Prominent nocturnal wheezing was present in eight of 12 children who were unaware of it. Since healthy children do not wheeze during sleep, the authors interpret the presence of nocturnal wheezes in asthmatic children as a manifestation of their disease. In these children, the degree of nocturnal wheezing correlated well with other asthma indices during treatment. Some asthmatic patients with severe airflow obstruction do not necessarily wheeze (silent lung) and wheeze monitoring may not be an adequate indicator of disease severity in these patients. However, the majority of asthmatics do wheeze, and nocturnal wheeze monitoring can be a practical noninvasive tool of monitoring disease severity and activity.

In the patients in this study, nocturnal wheezing was a prominent finding, whereas little or no wheezing occurred during daytime. Therefore, nocturnal ARM provides additional practical information that can help in monitoring the patient's asthma and the response to therapy.

Many characteristics of wheezes, such as amplitude, frequency range, number of simultaneous wheezes, duration and chest distribution, can be recorded and measured, but the parameter that best correlates with other clinical indices of asthma severity is wheeze duration [14]. In the present study, the authors used the total duration of wheeze activity, irrespective of the respiratory phase or site, as the primary quantitative measure of wheeze activity. Compared with previous studies $[18,19]$, the authors used a wider frequency range to identify wheezes so that low frequency wheezes and rhonchi, as well as high frequency tracheal whistles, were included in the wheeze range calculation.

In the current study, as in previous reports, the authors found wheezes in both the inspiratory and expiratory phases. What, if any, correlation exists between wheeze frequency and/or their predominant timing to the severity and anatomic distribution of airway constriction requires further research.

In order to provide a clinically meaningful output, the nocturnal wheeze activity is reported as a trend plot of $\mathrm{Wz}^{\%} \%$ in 30-s segments throughout the night, as illustrated in figure 2. A more condensed view is provided by the histogram (fig. 3), from which a numeric score (NWI) is derived, which quantifies the total nocturnal wheeze activity. The trend plot is also useful for identifying the timing and distribution of wheeze activity during the night; in some patients the wheezes were most prominent in the early morning hours, whereas in others, wheezes were present throughout the night. Some patients had mild wheezing throughout the whole night, while in others there were a few short episodes of high $\mathrm{Wz} \%$. It is clear that while both may have identical NWI, the distribution during the night on the one hand and the peak $\mathrm{Wz}^{\%}$ on the other, reflect different temporal patterns of airway constriction that may have different implications regarding aetiology, clinical course and treatment strategy.

The dropout rate in the current study (four of 16 originally enrolled), though significant, is not unusual for this type of study. It was a long and time-consuming study that required considerable commitment on the part of the subjects and their parents. Another limitation of this study is the small number of subjects. This number is adequate, however, for a pilot 
study, and to ensure appropriate statistical validity, the authors used more stringent nonparametric statistics, which demonstrated statistically significant changes. There was also no true control group who received placebo and followed longitudinally. This study was not designed as a therapeutic trial, but was meant to assess the utility of nocturnal wheeze monitoring in the assessment of asthma. Montelukast was given to create changes in asthma activity that can be followed and monitored, both conventionally and by ARM.

The authors found a substantial and statistically significant decline in wheeze activity and early symptom relief with montelukast treatment: $75 \%$ of the patients had a marked drop in nocturnal wheezing, while the rest had no significant response (fig. 4). A similar ratio of responders to nonresponders during montelukast treatment has been reported in other studies [20]. The wheeze activity during treatment correlated significantly $(\mathrm{p}<0.014)$ with baseline FEV1 and with PC20 $(\mathrm{p}<0.014)$ during treatment. The decrease in symptoms was associated with a parallel reduction in NWI.

The present study demonstrates the usefulness of nocturnal wheeze monitoring in the assessment of asthma patients. An unsuspectedly high level of nocturnal wheezing in the group of untreated asthmatic children, including those with mild asthma and normal spirometry, was found. Quantitative wheeze assessments, expressed as Wheeze Rate and Nocturnal Wheeze Index, corresponded well with changes in forced expiratory volume in one second, provocation dose where forced expiratory volume in one second dropped $20 \%$ below baseline level, and symptom scores. Thus, nocturnal wheeze monitoring and quantification provides a simple and noninvasive method for assessment of asthma severity and response to treatment. While this study was carried out in older children, it seems likely that monitoring of younger children, infants and elderly patients may be even more clinically useful by providing continuous objective measurements of asthma activity and response to treatment in patients who cannot perform spirometry.

Acknowledgements. The authors wish to acknowledge the skilful technical assistance of KMAT clinical coordinators. They thank S. Godfrey, D.W. Cugell and C.S. Irving for discussing the data and for their numerous, useful comments and suggestions.

\section{References}

1. Turner-Warwick M. Epidemiology of nocturnal asthma. Am J Med 1988; 85: 6-8.

2. Meijer GG, Postma DS, Wempe JB, Gerritsen J, Knol K, van Aalderen WM. Frequency of nocturnal symptoms in asthmatic children attending a hospital outpatient clinic. Eur Respir J 1995; 8: 2076-2080.

3. Stores G, Ellis AJ, Wiggs L, Crawford C, Thomson A. Sleep and psychological disturbance in nocturnal asthma. Arch Dis Child 1998; 78: 413-419.

4. Weersink EJ, van Zomeren EH, Koeter GH, Postma DS.
Treatment of nocturnal airway obstruction improves daytime cognitive performance in asthmatics. Am J Respir Crit Care Med 1997; 156: 1144-1150.

5. Morgan AD, Rhind GB, Connaughton JJ, Catterall JR, Shapiro CM, Douglas NJ. Breathing patterns during sleep in patients with nocturnal asthma. Thorax 1987; 42: 600603.

6. Ballard RD, Saathoff MC, Patel DK, Kelly PL, Martin RJ. Effect of sleep on nocturnal bronchoconstriction and ventilatory patterns in asthmatics. J Appl Physiol 1989; 67: 243-249.

7. Falconer A, Oldman C, Helms P. Poor agreement between reported and recorded nocturnal cough in asthma. Pediatr Pulmonol 1993; 15: 209-211.

8. Kercsmar CM. Asthma. In: Chernick V, Boat TF, eds Disorders of the Respiratory Tract in Children. Philadelphia, Saunders Co., 1999; p. 688.

9. Lenclud C, Cuttitta G, Visconti A, van Muylem A, Bellia V, Yernault JC. All night on line monitoring of wheezing: comparison with monitoring of airway resistance in nocturnal asthma. Bull Eur Physiopathol Respir 1986; 22: 191-199.

10. Kiyokawa H, Yonemaru M, Horie S, Kasuga I, Ichinose Y, Toyama K. Detection of nocturnal wheezing in bronchial asthma using intermittent sleep tracheal sounds recording. Respirology 1999; 4: 37-45.

11. National Institute of Health. Global Initiative for Asthma: (GINA) - the new GINA workshop report 2002. National Heart Lung and Blood Institute/World Health Organization Workshop Report. Bethesda, MD, National Institute of Health, 2002.

12. Gavriely N. Breath Sounds Methodology. Boca Raton, CRC Press, 1995; pp. 55-97.

13. Earis JE, Sovijarvi ARA, Venderschoot J. European Respiratory Society Task Force Report: Computerized Respiratory sound analysis (CORSA): recommended standards for terms and techniques. Eur Respir Rev 2000; 10: 585-649.

14. Baughman RP, Loudon RG. Lung sound analysis for continuous evaluation of airflow obstruction in asthma. Chest 1985; 88: 364-368.

15. Lim S, Gavriely N, Irving CS, Chung KF, Barnes PJ. Quantitative documentation of wheezes in asthma clinics using an automatic wheeze quantification device. Eur Respir $J$ 1999; 14: Suppl. 30, 147.

16. Gavriely N, Irving CS. Tracheal whistles - a new physical sign of airway constriction. Eur Respir J 1999; 14: Suppl. 30, 72 .

17. Avital A, Picard E, Uwyyed K, Springer C. Comparison of adenosine 5'-monophosphate and methacholine for the differentiation of asthma from chronic airway diseases with the use of the auscultative method in very young children. J Pediatr 1995; 127: 438-440.

18. Fenton TR, Pasterkamp H, Tal A, Chernick V. Automated spectral characterization of wheezing in asthmatic children. IEEE Trans Biomed Eng 1985; 32: 50-55.

19. Gavriely N, Palti Y, Alroy G, Grotberg JB. Measurement and theory of wheezing breath sounds. J Appl Physiol 1984; 57: 481-492.

20. Knorr B, Matz J, Bernstein JA, et al. Montelukast for chronic asthma in 6 to 14 year old children: a randomized, double-blind trial. JAMA 1998; 279: 1181-1186. 